\title{
Using a Groupware Space for Distributed Requirements Engineering
}

\author{
Daniela Herlea and Saul Greenberg \\ Department of Computer Science \\ University of Calgary \\ Calgary, Alberta, Canada, T2N 1N4 \\ danah,saul@cpsc.ucalgary.ca
}

\begin{abstract}
Requirements engineering (RE) is a necessary part of the software development process, as it helps customers and designers identify necessary system requirements. If these stakeholders are separated by distance, we argue that a distributed groupware environment supporting a cooperative requirements engineering process must be supplied that allows them to negotiate software requirements. Such a groupware environment must support aspects of joint work relevant to requirements negotiation: synchronous and asynchronous collaboration, telepresence, and teledata. It should also add explicit support for a structured RE process, which includes the team's ability to discuss multiple perspectives during requirements acquisition and traceability. We chose the TeamWave software platform as an environment that supplied the basic collaboration capabilities, and tailored it to fit the specific needs of RE.
\end{abstract}

\section{Distributed Requirements Engineering}

The misidentification of requirements is one of the most significant sources of customer dissatisfaction with delivered systems [13]. Consequently, development teams often include a requirements engineering process (RE) within a software development cycle to help them determine what needs to be designed.

The problem is that software stakeholders can work in environments that cross organizational and national boundaries, where team members and sub-groups may find themselves isolated from one another by distance and by time. One solution to this problem is to have a networked team use groupware specifically designed to support a distributed RE process.

In this paper, we consider ways that groupware can support how a distributed team gathers requirements through a negotiated RE process. The naïve view of $R E$ is that requirements are immutable facts about a situation that exist a priori and independent of the client's perception [4]. Our view is that requirements gathering goes beyond the one-way transfer of information from clients to designers. Rather, requirements are a negotiated product generated through a collaborative requirements process, where both clients and designers socially construct their requirements [4,14]. Consequently, groupware for RE must support a truly collaborative requirements negotiation process.

Groupware should support activities identified as major problems in $\mathrm{RE}$, including domain knowledge acquisition and traceability [16]. The issue here is that the participants in the process have multiple perspectives over the system to be designed [5], and that communication in RE "is much more about developing an understanding of each other's views and perspectives, about sharing knowledge and about learning as a result of a shared experience" [14]. Consequently, we focus on the ability of team members to express, discuss and negotiate perspectives during requirements acquisition and traceability.

We begin the paper by describing general groupware design requirements that also apply to an RE process. We then explain why we chose the TeamWave Workplace product $[10,20]$ as our base technology. Next, we discuss how TeamWave was customized to support particular tasks within distributed RE, and we offer a scenario of use to illustrate system's capabilities. The paper closes by outlining further research directions we believe are necessary for the design of groupware that fully supports negotiative requirements engineering processes.

\section{Groupware Requirements for Remote Requirements Negotiation}

While groupware for distributed collaboration is well studied in the area of Computer Supported Cooperative Work (CSCW) [7,9], little attention has been given to how it can support the RE process. Still, several groupware design factors are highly relevant to requirements negotiation: support for both asynchronous and synchronous collaboration, telepresence, teledata, and process support.

Through asynchronous collaboration [7,9], team members can construct requirements individually and contribute them to the collective activity of the group for later discussion. This is especially important when groups are distributed across time zones and when real time meetings are hard to schedule. Through asynchronous capabilities, individuals will be able to access all 
available requirements documents and artifacts for review, modification, and/or annotation. Asynchronous collaboration is also important for group coordination, including setting up meetings, sending out reminders, tracking schedules, and so on [7,9].

Most existing groupware RE systems now concentrate on asynchronous collaboration. For example, a number of prototypes presented in the Proceedings of WET ICE 96 offer asynchronous web support for requirements elicitation, analysis and validation e.g., WHERE [6] and GBRAT [2].

Synchronous collaboration [7,10] lets distanceseparated people work together at the same time. Our opinion is that the wholly asynchronous systems mentioned previously are inadequate, as we believe that real time collaboration and discussions are a necessary component of the group RE session. Specification of requirements is regarded as a knowledge acquisition task, and conversation is the main vehicle for gathering, clarifying and validating the knowledge about requirements [5]. Thus synchronous communication as well as access to a shared workspace is important in RE, as detailed below.

Telepresence is defined as a "way of giving distributed participants a feeling that they are in the same meeting room" [10]. In RE, as in any negotiation meeting, a sense of presence is necessary to help participants mediate and coordinate their real time discussions, and to achieve a common understanding of the work process [14]. Presence is also important in helping a group create and manipulate the meeting artifacts - the shared requirements representations-that arise during the meeting activities. These artifacts are often the focus of a group's discussion, negotiation, and decision-making activities.

Teledata is a "way of having participants bring into the meeting the materials and on going work they wish to share with others" [10]. In RE, issues of concern include the ability to generate and share requirements representations such as text and graphics. The group needs facilities to support the input, storage, access and retrieval of information about requirements (e.g. results of brainstorming, lists of important items and decisions) [14]. Bringing requirements documents or other work artifacts in the shared workspace is essential during discussions of requirements representations and perspectives, traceability, and validation.

Process support. Because requirements engineering often follows a structured process (e.g., JAD), the system should support how a group moves through the process. While there are many process-oriented decision support systems [15], these do not reflect activities specific to RE. We believe that groupware should provide enough structure to guide a group through the particulars of RE, both to help them stay on track and to remove the tedium of recreating routine structures.

While there are many more design factors, we believe that these five general requirements can help support a distributed RE processes by providing mechanisms for designers to "get to know" the clients [14], and to afford communication between the analyst and the clients.

\section{TeamWave Workplace}

We chose TeamWave Workplace [10,20] as a groupware system that can support the basic needs of a distributed RE group. This section briefly describes the system, while the following section describes how it can be customized to support activities particular to RE.

TeamWave implements a room-based metaphor. Rooms can be created by participants, and each room can be stocked with any number of collaborative tools. Tools include:

- ever-present teledata facilities such as a shared whiteboard which occupies the "walls" of the room

- application-specific teledata tools such as groupware post-it notes, bulletin boards, note organizers, brainstorming and voting tools, file viewers, data bases, action item organizers, and many more.

- ever-present telepresence facilities, including a textbased chat tool for typing short messages to other occupants of a room, a list of other people in the room, telepointers showing where others are gesturing, etc.

For example, Figures 1 and 2 illustrate two different rooms created for RE. The back 'wall' of each room is a whiteboard, and Figure 1 shows some text and graphics drawn on it. The smaller windows within the rooms are various tools. Figure 2 also shows a list of the room's occupants on the left side.

Through its rooms metaphor, TeamWave combines the richness of specialized real-time groupware applications with a virtual environment that provides a persistent working and meeting space. It is 'teamware', as it has a strong notion of supporting small communities. A team can form a customized (and secure) environment by creating rooms, by shaping their contents, and by mediating access to items by particular group members. Because it is cross platform (it runs on PC, Unix and Mac machines), it can serve teams that use different technologies.

TeamWave supports real time conferencing. When participants from remote sites enter a common room, they can immediately sense each other, communicate via chat (as audio is not part of TeamWave, it must be done via another tool), and use its collaborative tools. TeamWave also supports asynchronous collaboration. Because all artifacts in a room are fully persistent, participants can enter a room at any time, do their work, and leave notes and work artifacts for others to view at a later time. 


\section{Customizing TeamWave to RE}

We have argued that TeamWave can support collaborative activities of a distributed group. In this section, we will describe how TeamWave was customized to support a structured RE process, as well as the team's ability to discuss multiple perspectives during requirements acquisition and traceability. We will use a scenario of a team negotiating requirements for a library system.

\subsection{Creating a meeting space that supports the requirements process}

Unlike many group decision support systems [15], the room metaphor portrayed by TeamWave does not impose any direct support for meeting processes. Nothing dictates which tools be used for which task, or how they should be used. This is not necessarily a problem in conventional real time groupware, as the social practice of the group can dictate what a room can be used for [10].

However, TeamWave rooms can be customized ahead of time by a facilitator to support particular processes, and modified on the fly to reflect the team's evolution through the process. The idea is to make virtual rooms and their tools act as a spatial setting for interaction that frames and structures appropriate behavior [12].

For example, the TeamWave 'Planner' room in Figure 1 was created by a facilitator to capture the major steps in a particular RE process. The 'diagram' in the room indicates an iterative process, where team members step through requirements discovery (upper left),

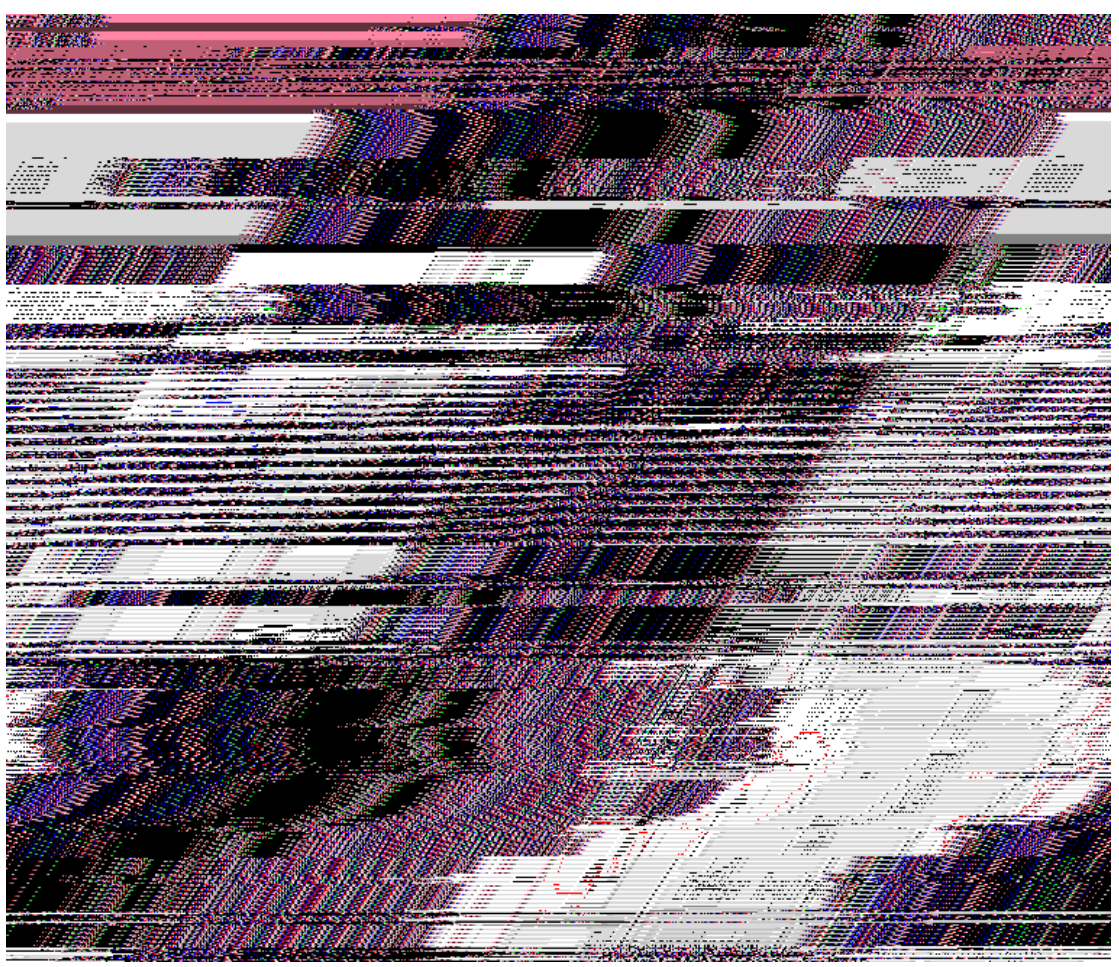

Figure 1: An Overview of an RE Process is provided in the Planner Room requirements refinement (upper right), perspectives analysis (lower right), and prototype validation/solution selection (lower left). In turn, each major step comprises several activities e.g., requirements discovery includes scope definition, brainstorming, and elicitation of enduser requirements (upper left). What makes this special is that this 'diagram' is actually a navigation space, where each icon is a doorway into another room that in turn has been prepared to support a particular activity. For example, if a team is ready for the scope definition step in requirements discovery, they would press that icon and enter a room configured for this particular sub-task. This new room is illustrated in Figure 2. As the group uses that (or other) rooms, the room itself will evolve to reflect their activities: documents created with the tools persist in the space (e.g., Figure 2 shows a partially created scope definition); new tools can be introduced; and new rooms can even be created. Finally, rooms can be fashioned that support tangential parts of the group process. For example, the central cluster of icons in Figure 1 are doorways into a generic meeting room, a 'coffee room' for casual talk, a 'Read Me' room that contains extra documentation that should be read by group members, and so on.

The collection of customized rooms provide a structure that guides the group along certain steps of the requirements process. However, it is important to realize that this is a highly flexible structure, as it does not force the group to go through each and every step in a particular order or manner.

\subsection{Expressing and negotiating multiple perspectives}

The expression of individual perspectives is a difficult process even in a face-to-face setting [6]. Requirements negotiation is influenced by the team members' use of different terminologies, their levels of domain knowledge, their organizational and political considerations, as well by their personalities $[1,18]$. The differences in perspectives become acute in a geographically distributed $\mathrm{RE}$ environment, as participants may have had little or no previous social interaction with one another that could have helped resolve these differences [12].

This section discusses the support TeamWave offers to the expression and the negotiation of multiple perspectives during activities such as requirements acquisition and traceability. In our discussion, a 
perspective is regarded as an explicit description of the world from a particular angle, and there is a relationship between perspectives and roles [5].

\subsubsection{Requirements acquisition}

We configured TeamWave to support participants' exchange of one another's views and perspectives during the requirements acquisition activity. In particular, we supplied a group with concept mapping tools and a note organizer. With the concept mapping tools, participants describe particular parts of their knowledge, where the concept map embodies a representation schema that participants can use to structure their viewpoints [5]. With the note organizers, participants specify their position that forms the basis behind their perspective.

Figure 2 illustrates this by example, where two participants, a librarian and a borrower, are involved in the scope definition phase of the RE process. They are in the Scope Definition Room, customized ahead of time with appropriate tools to help them define the concept of a 'book'. We see two instances of the concept map tool, each used to express the notion of a book from two different perspectives. We also see how they have specified their roles along with their argumentation for each representation. The spatial properties of a room also means that the participants can structure and annotate the space, simply by moving relevant work artifacts close to one another. For example, the role/argument text was placed to partially overlap the concept map that it is related to.

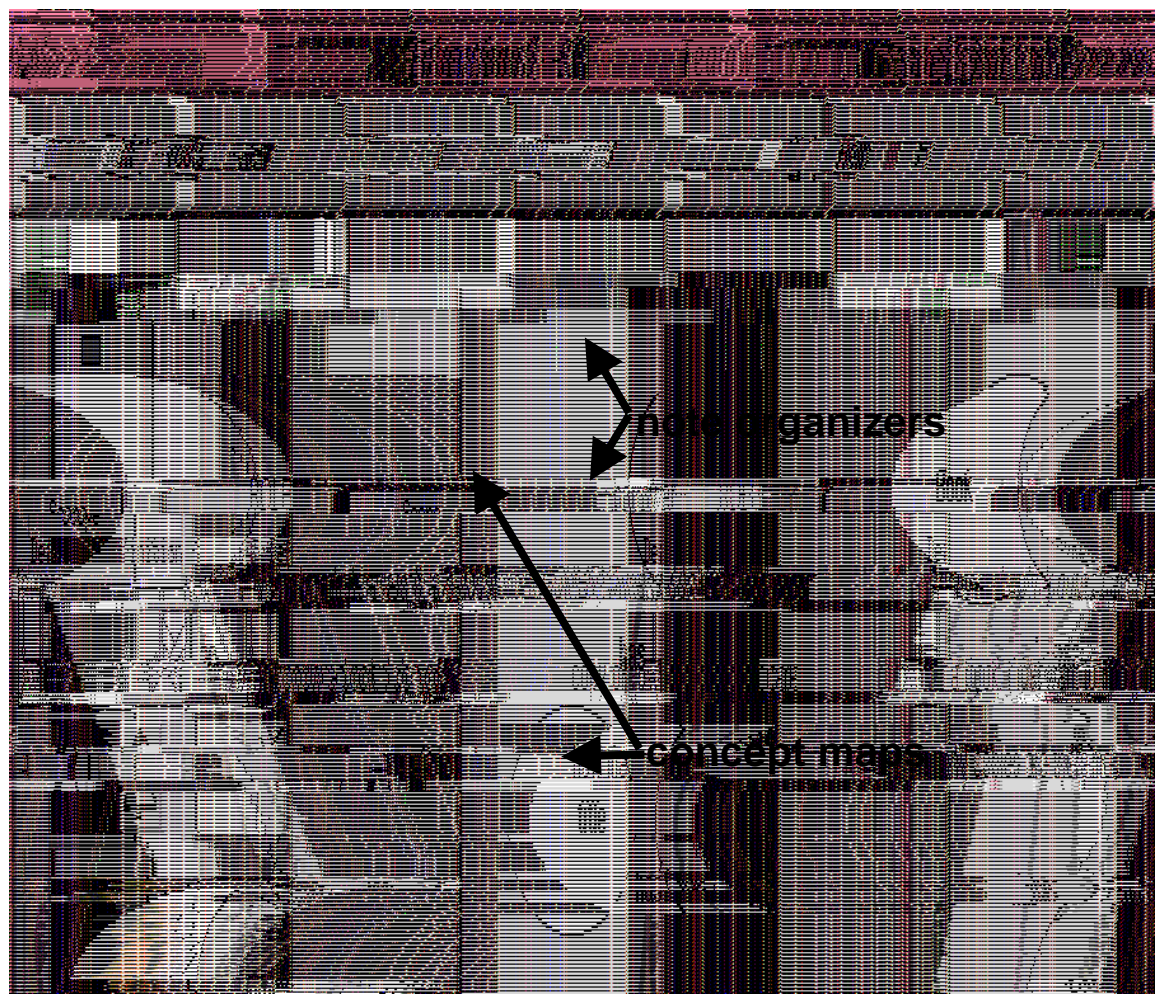

Figure 2: Scope Definition Room-Individuals specify perspectives and roles
We believe that it is important that the virtual environment enables not only the description of several perspectives, but that it also fosters the development of mutually comprehensible requirements representations through real time negotiation and exchange. In particular, the concept maps in Figure 2 may have been created asynchronously. However, both participants could agree to meet in real time, where they would examine, clarify and debate the captured perspectives. TeamWave has facilities that make real time interaction with teledata both reasonable and effective. People can simultaneously edit or modify requirements representations in a room, and see each other doing so. They can also gesture to items in a room through telepointers, one for each user, which act as surrogates for the participant's hand gestures in the shared workspace. Other tools could be imported to assist this process: outlining tools to create hierarchical structures; concept maps to analyze relationships between data and users; voting tools to identify priorities and evaluate options and so on.

\subsubsection{Collaborative requirements traceability}

The process of requirements acquisition can never be regarded as complete during the lifecycle of a software system [5]. Consequently, software engineers often have to retrace how requirements arose and the logic behind them during the process of generating the requirements specification. Engineers use requirements traceability techniques to determine if the software requirements have been implemented in the system [3]. During software development, they should be able to trace requirements backwards from design components to their motivating requirements [8], and to find the originator and the rationale for these requirements (this will be illustrated shortly with an example). They do this activity to check their understanding of requirements, and to analyze the impact in case of documentation changes [3]. Because it is part of requirements negotiation, the changes made are compliant with people's concerns, and should be mutually consistent [19].

The first issue relevant to groupware is how the system maintains repositories of requirements traces. TeamWave provides this 'for free' through its persistent space and its versioning tools. The fully persistent workspace means that items left in a room stay there, and that the room's content always contained that last snapshot of the group's activity. While conventional file systems require the engineer to find relevant 
files and to reconstruct their relations to one another, TeamWave users just have to enter a room to see all items and how they relate to one another. TeamWave also maintains a version repository of all rooms and their contents. This means that previous versions of requirements representations-lists, definitions, drawings and diagrams - can be retrieved at any time. Consequently, one can monitor the history of how the team manipulated its artifacts in that room, and can see how a group progressed from a "personal view" over a particular requirements or situation to a "common view" as consensus is reached.

The second issue relevant to groupware is how the group can collaborate during the requirements traceability activity. This is achieved by allowing the team to structure a room in a way that brings together the participants and the artifacts required for tracing particular requirements. This is best illustrated by an example, where we will see how a group could have configured a TeamWave room to help them trace how well an interface prototype design matches its requirements specifications.
Figure 3 illustrates the example. The group enters this "Worth Proceeding" room from a doorway that was part of the "Validating the prototype" step in Figure 1. The room brings together the design artifacts and requirements specification. In particular, it contains:

- a screen snapshot of a 'lending transaction' interface prototype, held by an image tool (top left);

- an informal and a semi-formal representation of the requirements, displayed as html documents presented in a groupware web browsing tool (bottom and right side respectively);

- original copies of these documents that can be imported to a local person's machine through a file holder tool (bottom left);

- a doorway into a related room (left side).

To create this room, the team used the image tool to display a screen snapshot of the prototype. They also primed the Web browser tools with entry pages into the requirements documents that had motivated the prototype: these documents were produced earlier in the $\mathrm{RE}$ process. The team collaborates by browsing and discussing the various requirements documents, and

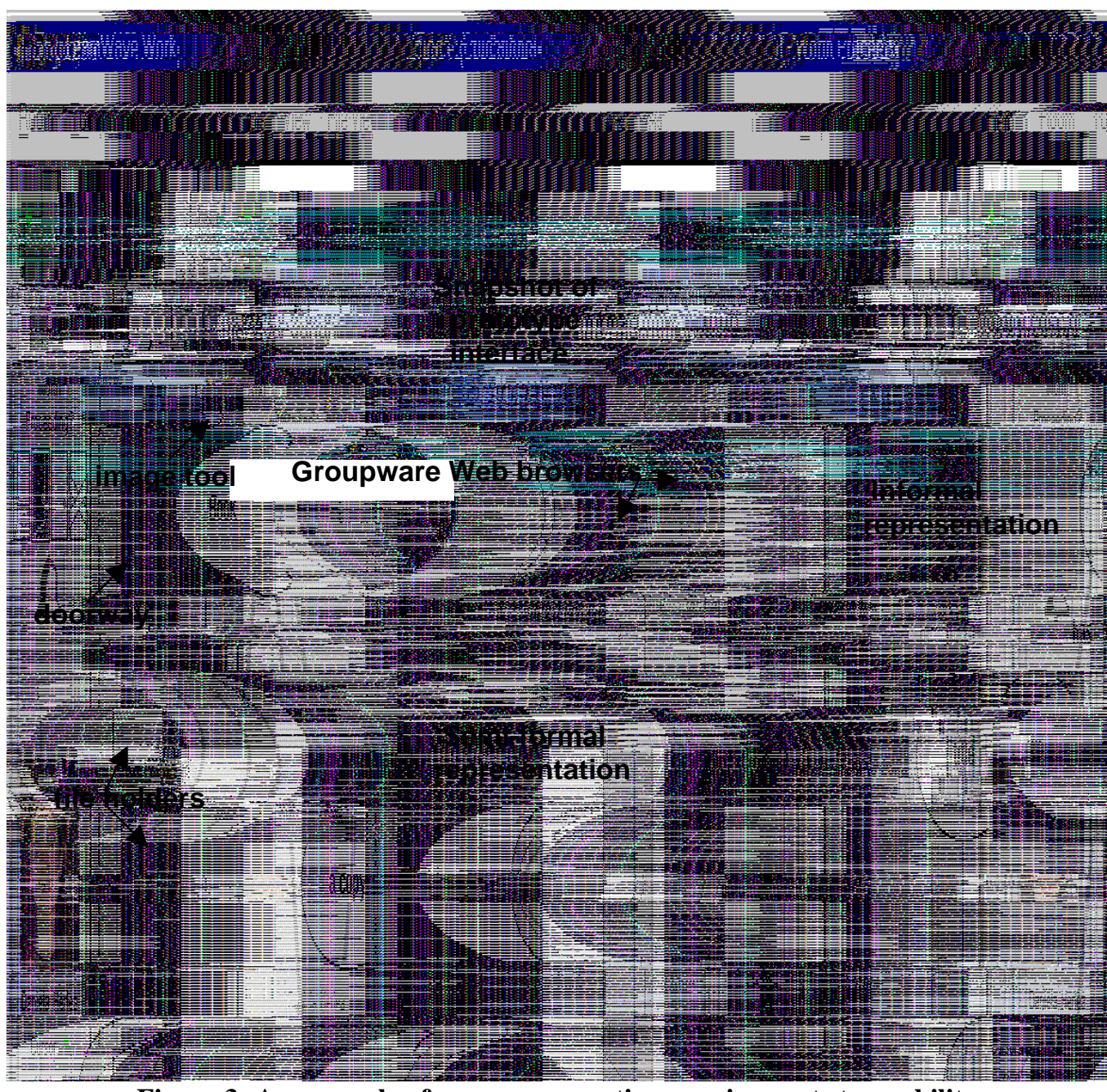

Figure 3: An example of a room supporting requirements traceability seeing how well the interface satisfies them. Following the hypertext links in the semi-formal document, the group could trace the representation of the concept 'book' (a component of this transaction): each entry in the semi formal document has links to its natural language requirement source and rationale, stored in the informal document. In the scenario shown in the figure, the links associated to the concept 'book' were followed and the two perspectives (containing the role and the argument of the originator) were traced. When conflicts, ambiguities and/or inconsistencies of the requirements specification appear, the team can analyze and negotiate these perspectives through TeamWave's standard collaborative tools. They 
can also discuss the impact of changing the features of the 'lending transaction' prototype and how it would reflect the various perspectives of the 'book' concept.

\section{Discussions and Conclusions}

In this paper, we argued that groupware should be developed that supports a distributed requirements engineering process. After outlining several general capabilities that groupware should provide, we illustrated by example how systems could support activities specific to RE. This included the creation of a groupware space supporting the overall RE process, as well as specific activities such as collaborative expression and negotiation of multiple perspectives. TeamWave was presented as an enabling technology that can be customized to handle these RE-specific activities.

We believe that this research represents only a start in understanding how groupware can support distributed requirements engineering. Further research, which should include studies of real work settings, are necessary before we can design groupware that fully supports requirements engineering as a negotiative process. Issues of concern that still have to be addressed include:

- Do people using the system acquire enough of a shared understanding of requirements and perspectives to progress effectively through the process?

- What other activities in the RE process must be supported explicitly by tailor-made groupware?

- Is a facilitator knowledgeable in the RE process necessary, and if so, how are their duties supported by the system?

- What organizational issues are specific to RE, and how will that affect how the system is introduced and adopted by the networked organization?

- How can we evaluate the effectiveness of the team using the system [11]? While we have discussed the scenario presented in this paper with software engineers and received generally positive feedback, this is a long ways from knowing that it will actually work in practice.

- How well does the system handle general collaboration activities, For example, can it support larger teams working together in real time? Does it scale up to teams that produce and manipulate large numbers of work artifacts? Is the telepresence supplied appropriate and sufficient for team cohesiveness? Where does the room metaphor break down? These are all basic CSCW issues, and progress in that field will influence how systems for RE are created.

We believe that systems should be developed that support distributed requirements engineering. The research in this paper provides a beginning. However, it must be remembered that requirements negotiation is a subtle and complex process that is still ill-understood even in small, co-located and cohesive software engineering teams. Understanding the nuances of this process is vital if effective groupware designs are to be developed.

\section{References}

[1] A. Al-Rawas and S. Easterbrook, "Communication problems in requirements engineering: a field study", Proc. of the First Westminster Conf. on Professional Awareness in Software Engineering, Royal Society, London 1996.

[2] A. Anton, E. Liang and R.A. Rodenstein, "A web-based requirements analysis tool", in [17]

[3] S.A. Bohner and R.S. Arnold, "An introduction to software change impact", in Software Change and Impact Analysis, IEEE Computer Society Press, Los Alamitos, California, 1996

[4] L. Brooks and M. Jones, "CSCW and requirements analysis: requirements as cooperation/requirements for cooperation, in P. Thomas (ed), CSCW Requirements and Evaluation, Springer-Verlag London, 1996

[5] S.M. Easterbrook, "Elicitation of requirements from multiple perspectives", $\mathrm{PhD}$ Thesis, Imperial College of Science Technology and Medicine, University of London, 1991.

[6] S.M. Easterbrook and Callahan, "Independent validation of specifications: a coordination headache", in [17]

[7] C.A. Ellis, S.J. Gibbs and G.L. Rein, "Groupware: some issues and experiences", Comm. of the ACM, January 9-23, 1991

[8] O.C.Z. Gotel and A.C.W. Finkelstein. "An analysis of the requirements traceability problem", Proc. of the $1^{\text {st }}$ Intl. Conf. on Requirements Engineering, Colorado Springs, 94101, 1992

[9] S. Greenberg, M. Roseman, D. Webster and R. Bohnet, "Human and technical factors of distributed group drawing tools", Interacting with Computers 4(1), 364-392, 1992

[10] S. Greenberg and M. Roseman, "Using a room-based metaphor to ease transitions in groupware", Report 98/611/02, Dept Computer Science, Univ. of Calgary, 1998

[11]J. Grudin, "Why CSCW applications fail: Problems in the design and evaluation of organizational interfaces. Proc. of CSCW'88, 85-93, Portland, Oregon, 1988

[12] D.E. Herlea, M.L.G. Shaw and B.R. Gaines, "Locales for requirements engineering", Report 98/610/01, Dept. Computer Science, Univ. of Calgary, AB, Canada, 1998

[13] L.A. Macaulay, "Requirements Engineering", SpringerVerlag London, 1996

[14] L.A. Macaulay, "Cooperation, requirements analysis and CSCW", in P. Thomas (ed), CSCW Requirements and Evaluation, Springer-Verlag London, 1996

[15] J. Nunamaker, A. Dennis, J. Valacich, D. Vogel and J. George, "Electronic meeting systems to support group work", Comm. of the ACM 34(7), 40-61, 1991

[16]C. Potts, "Working group report on requirements engineering in and for networked enterprises", in [17]

[17] Proceedings of WET ICE'96, June 1996

[18] M.L.G. Shaw and B.R. Gaines, "Knowledge acquisition: some foundations, manual methods and future trends", Proc. of the 3rd EKAW, Paris, 1989

[19] I. Sommerville and P. Sawyer, "Requirements Engineering: A good practice guide", Wiley, 1996.

[20] TeamWave Software Ltd., http://www.teamwave.com/ 